\title{
Three-Dimensional Modelling of Proton Ingestion Episodes in Low-Mass Stars
}

Stuart A. Heap*

Monash University

E-mail: stuart.heap@monash.edu

Richard J. Stancliffe

Monash University, Australian National University

John C. Lattanzio

Monash University

David S. P. Dearborn

Lawrence Livermore National Laboratory

\begin{abstract}
We have modelled a dual shell flash (DSF) in a low-metallicity $1.5 \mathrm{M}_{\odot}$ AGB star using the 3dimensional hydrodynamic program "Djehuty", observing how the evolution of these events compares to 1-dimensional models, which are hypothesised to be inaccurate due to the simplifications in the treatment of convective processes. In particular, the stability of the separated convective structure following hydrogen ingestion is investigated. In both models constructed, the split convective zone structure was found to be unstable, with the velocities within the inner convective zone increasing until material breaks through the gap and recombines the two regions into a large single convective region.
\end{abstract}

XII International Symposium on Nuclei in the Cosmos

August 5-12, 2012

Cairns, Australia

${ }^{*}$ Speaker. 


\section{Introduction}

Models of sufficiently low-mass and low-metallicity stars consistently undergo an event known as a proton ingestion episode (PIE) during the core or shell helium flash $[1,2]$. In 1D models this event involves the convective region splitting into two - with the upper region being powered by the burning of hydrogen, and the lower by that of helium [3]. The material in the upper region is eventually mixed into the envelope, while the material in the lower region remains internal.

Previous multi-dimensional studies have indicated that the splitting of this convective zone may be unstable when full 3D hydrodynamics is used rather than 1D mixing length theory [4]. We investigate the evolution of a split convective zone in a fully spherical, three dimensional hydrodynamic model, using "Djehuty"[5]. This is the first fully spherical model with nuclear physics to be made of a star with a split convective region.

Two models have been constructed with approximately $1.98 \times 10^{6}$ cells. The first, named CoreRes, has resolution concentrated on the centre, while the second model, named GapRes, has approximately a factor of 3 improved radial resolution in the region of the gap between the convective regions, while maintaining the same angular resolution. For an initial model, both of these used a $1 \mathrm{D}$ model of a $1.5 \mathrm{M}_{\odot}, Z=1 \times 10^{-5}$ star, the convective region of which has just split during a thermal pulse. This model was created with the STARS code [6].

\section{Results}

In both models, faster convective motion results in the hydrogen burning shell rapidly relocating to a much deeper level, very close to the helium burning region. Without the energy released by this burning, the temperature inversion which was supporting the split was rapidly erased. With the removal of this temperature inversion, fast moving plumes of material are able to pass over the gap uninhibited, and the gap between convective regions is destroyed. This is seen to occur in a little over an hour in CoreRes, and approximately seven hours in GapRes. The longer timescale for the breakdown of GapRes' gap occurs because the lower resolution of CoreRes results in more hydrogen being ingested, bringing more fuel to the burning region, resulting in faster convective motion.

Figure 1 shows the loss of the temperature inversion maintaining the gap, while figures 2 and 3 both show the evolution of the convective velocities as a result of this change to the temperature structure. The gap between convective regions is seen as a local minimum in figure 2, located at approximately $4 \times 10^{9} \mathrm{~cm}$. It is seen to reduce in depth and move outwards with time. Figure 3 shows that at earlier times there is a visible gap between the convective zones, where it is seen as a narrow region of white between two layers of colour. Over time this gap reduces in magnitude, and is eventually destroyed.

Also observable in figure 2 is that the velocity profile is qualitatively different from that seen in 1D studies. First of all, in the 1D models, the outer convective region is considerably faster than the inner convective region, while in our 3D models, the inner region is generally the faster moving one, and differences between the two regions are generally much smaller. Secondly, the gap between the regions is much smaller in the 1D models than in 3D, in 1D the gap is $8.4 \times 10^{7} \mathrm{~cm}$ wide. The mesh spacing of CoreRes and GapRes were $1.5 \times 10^{8} \mathrm{~cm}$ and $6 \times 10^{7} \mathrm{~cm}$ respectively. As 

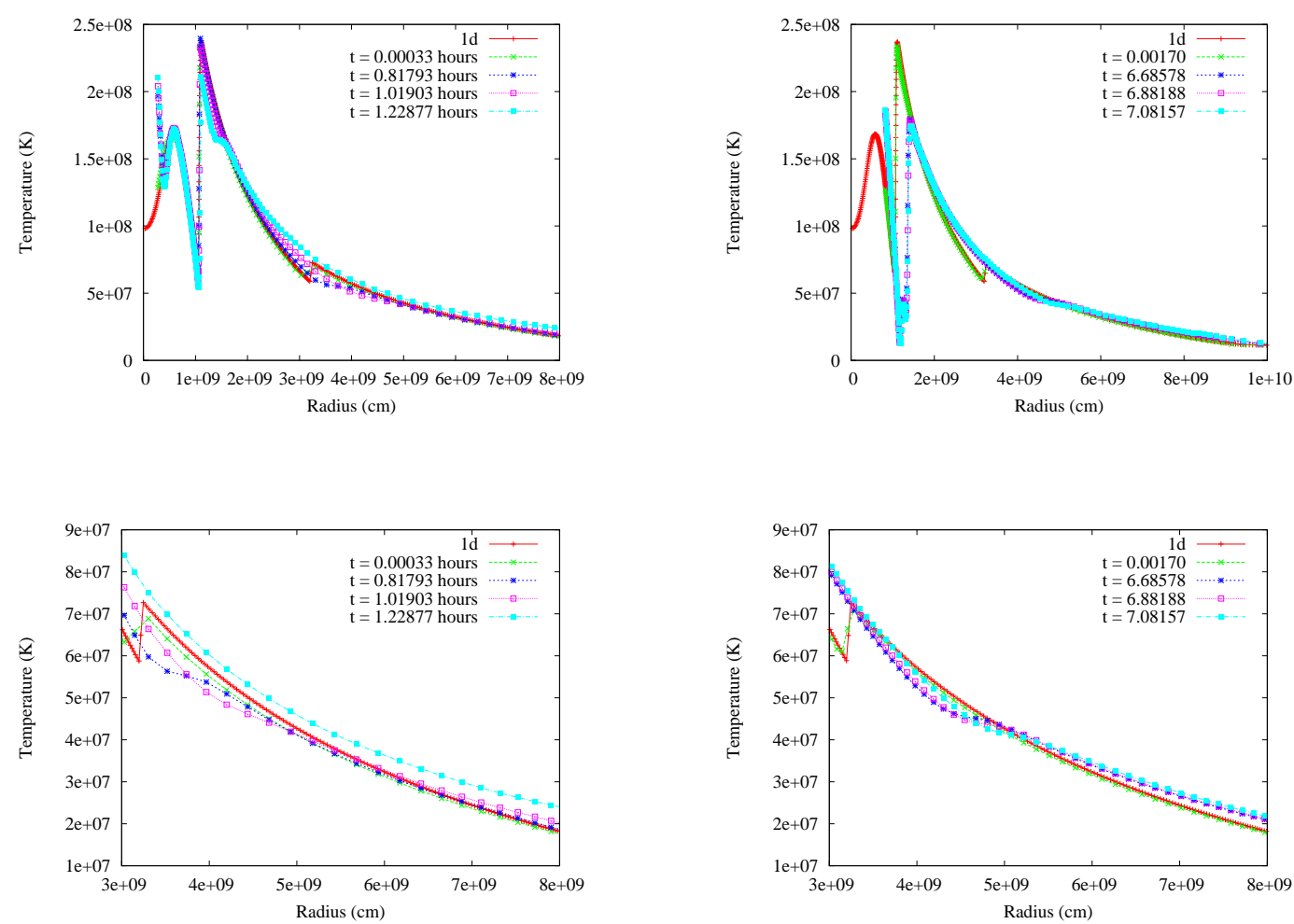

Figure 1: Temperature against radius for the two models, including a comparison to the one-dimensional input model. The lower images are zoomed in on the temperature inversion which maintains the gap between convective regions.

such, CoreRes would not be expected to be able to resolve the gap as it is seen in the 1D models, but it should be just resolvable by GapRes. The gap in 3D also shows a non-zero velocity. The movement in this region is believed to be caused by gravity waves generated by the neighbouring convective regions.

It is possible that the breakthrough of the gap is an artefact of the relatively low resolution used in this study. However, as each model has the resolution concentrated in a different region, and both ultimately show the same behaviour of the gap breaking down, we are reasonably confident that the destruction of the gap is real.

\section{Conclusion}

The split between convective zones was found to be unstable in three dimensions, suggesting that the results found in 1D models are an artefact of the simplifications of convection used. All current estimates of the surface abundance and yields of low- and intermediate-mass population II and III stars are based on the findings of 1D models The results here tentatively show that these predictions may be inaccurate. There is some evidence that population I stars may undergo PIEs [4], which would also call yields of these stars into question. Higher resolution models (which we are currently preparing) need to be constructed to be confident in these findings, but if they are 

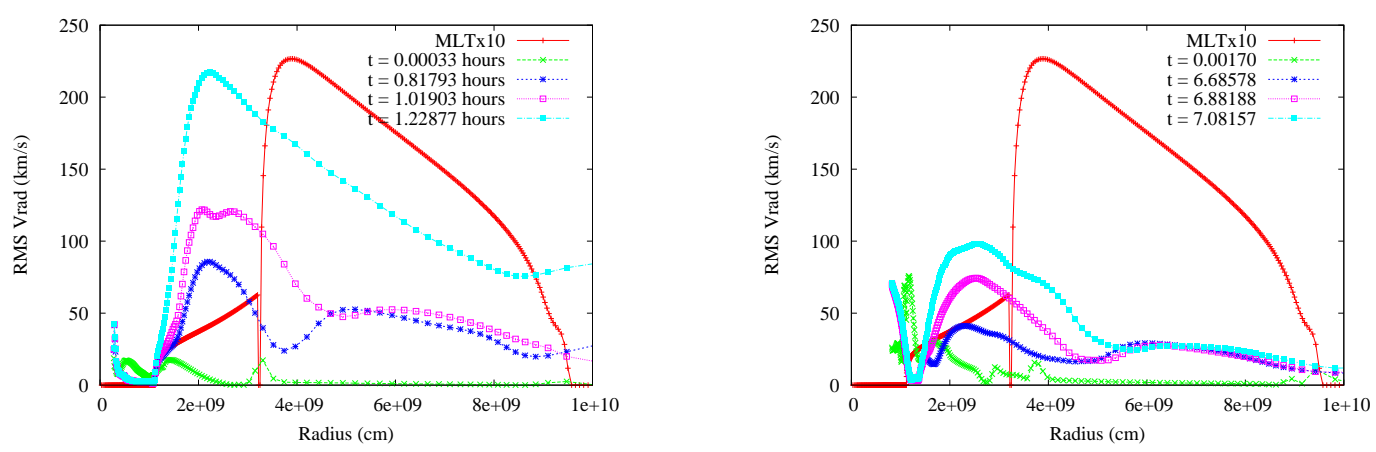

Figure 2: Spherically averaged RMS velocity as a function of radius throughout both models. Note that the 1D MLT velocities have been multiplied by 10 for easier comparison.

found to be accurate, it would indicate that the treatment of convection used in these 1D models is inadequate for these events. The resolution used in these studies is significantly lower than we would like, and we do not have sufficient evidence to claim that these models have converged. The higher resolution models which we are preparing may show qualitatively different results.

We are currently developing a two-stream advective mixing algorithm for use in the 1D evolution code "MONSTAR", which we believe will be more suitable than the currently used diffusive mixing algorithms to model these events. A two stream algorithm will be able to realise more of the intricacies of convective motion, particularly the fact that downflows generally move faster than upflows, resulting in the ingested material being transported further before burning.

\section{References}

[1] M. Y. Fujimoto, I. Iben Jr and D. Hollowell Helium flashes and hydrogen mixing in low-mass population III stars, The Astrophysical Journal 349 (1990) 580.

[2] S. Cassisi, V. Castellani and A. Tornambe, The evolutionary properties and peculiar thermal pulses of metal-deficient low-mass stars, The Astrophysical Journal 459 (1996) 298.

[3] D. Hollowell, I. Iben Jr and M. Y. Fujimoto, Hydrogen burning and dredge-up during the major core helium flash in a $Z=0$ star, The Astrophysical Journal 351 (1990) 245.

[4] M. Mocak, S. W. Campbell, E. Muller and K. Kifonidis, The core helium flash revisited III. From pop I to pop III stars, Astronomy and Astrophysics 520 (2010) A114 [1003.3646].

[5] D. S. P. Dearborn, J. C. Lattanzio and P. P. Eggleton, Three-Dimensional Numerical Experimentation on the Core Helium Flash of Low-Mass Red Giants, The Astrophysical Journal 639 (2006) 405 [astro-ph/0512049].

[6] R. J. Stancliffe and J. J. Eldridge, Modelling the binary progenitor of Supernova 1993J, Monthly Notices of the Royal Astronomical Society 396 (2009) 1699 [0 904 . 0282]. 

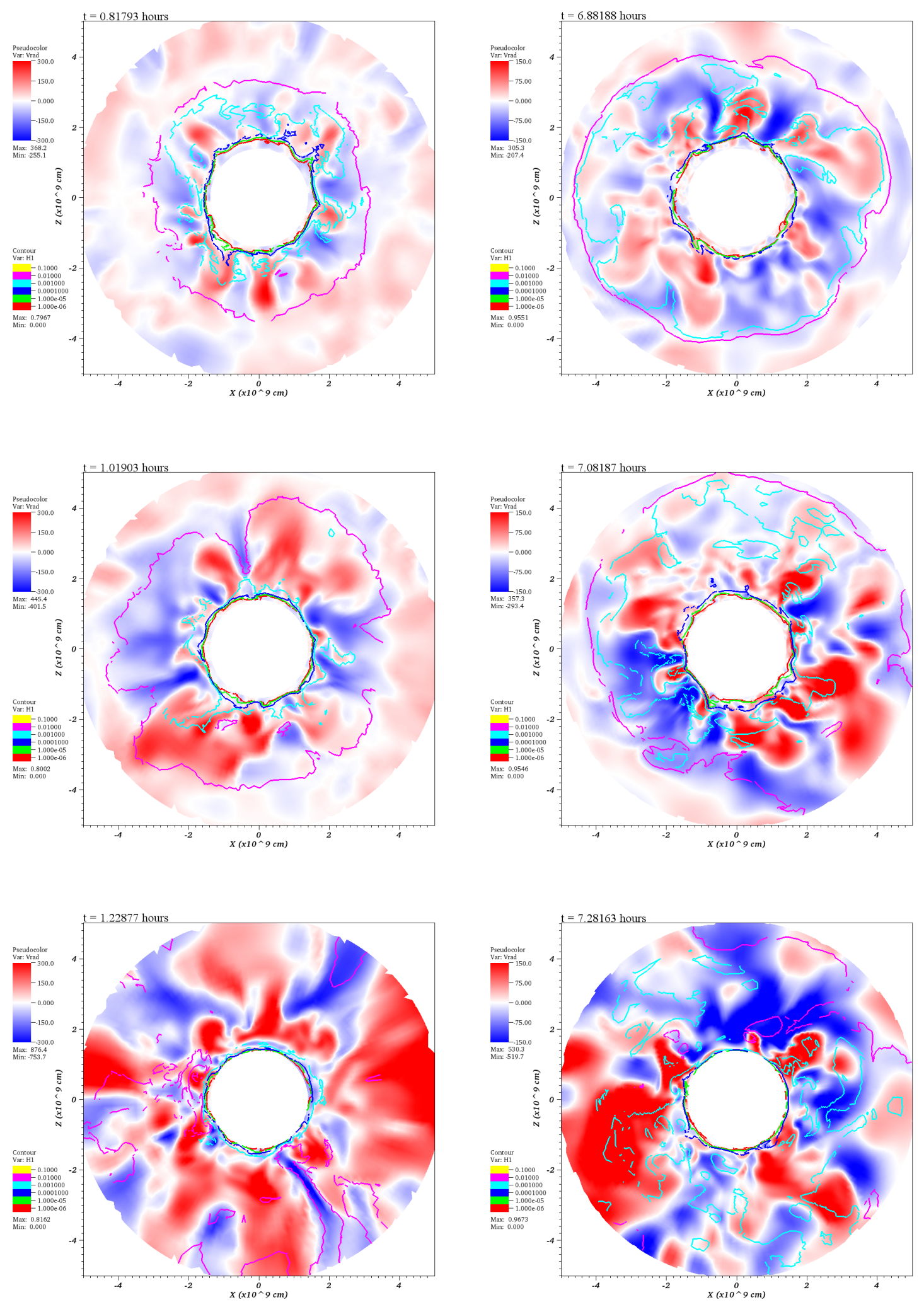

Figure 3: Slices of the two models in the $X-Z$ plane. Colour indicates radial velocity, and contours are hydrogen abundance. Left images are CoreRes, right are GapRes 